\title{
A Two Delay Differential Equations Model of Blood Partial Pressures in Human Cardiovascular-Respiratory System
}

\author{
Jean Marie NTAGANDA
}

\author{
University of Rwanda \\ College of Science and Technology, School Science, \\ Department of Mathematics, Huye Campus, Rwanda \\ jmnta@yahoo.fr, j.m.ntaganda@nur.ac.rw
}

\begin{abstract}
In [1], a mathematical model is proposed. It is an ODE model of Blood Partial Pressures in Human Cardiovascular-Respiratory System. This model consists of two components: systemic arterial compartment and systemic venous compartment. This model has been important in the field of mathematical modeling of Cardiovascular-Respiratory System. We introduce a time delays to the model to describe the time between controls (Heart rate and alveolar ventilation) of cardiovascular-respiratory and the pressures (systemic arterial and systemic venous pressures) during physical activity. The numerical simulation has been done to test the response of controls (Heart rate and alveolar ventilation) of cardiovascular-respiratory system to variation of trajectories of pressures.
\end{abstract}

Keywords: Systemic arterial pressure, Systemic venous, Heart rate, Alveolar ventilation, delay, numerical simulation.

\section{INTRODUCTION}

The cardiovascular system plays a crucial role in transport of oxygen, carbon dioxide [2] and nutrients through the blood from the various muscles and organs. The gases (oxygen and carbon dioxide) between the environment and the tissues of human body are transported by the respiratory system through the blood flow [3], [4]. A very important discussion for human health is the control of the cardiovascular and respiratory system. The improvement of diagnostics and treatment of diseases for this system are based on a good knowledge of its control mechanism where the autonomic nervous system controls and regulates all activities. In terms of control function in cardiovascular system; the autonomic nervous system controls and regulates all activities. The heart rate is controlled by both systems (sympathetic and parasympathetic nervous systems) [5] [6]. If the sympathetic nervous system excites a particular organ, often parasympathetic nervous system inhibits it [7].

The mathematical models exist for different domain of medicine including epidemiology, immunology, physiology, cell mobility. Many of these mathematical models focus on the applications in controlling the human body. Since the 1950's, the mathematical models for this system have been developed using dynamical mathematical models. Most of them arise from the compartmental theory [1], [5], [8], [9], [10], [11], [12], and [13]. The mathematical models incorporating the transport delays have been proposed [15]. It was noticed that the heart-lung interaction is inherently unstable, especially if certain heart-lung disease or injuries are present. For realistic contribution, the proposed models can be employed for controller synthesis for medical equipments. In some mathematical model, delay is introduced into the control respiratory system due to the physical distance which gases (oxygen and carbon dioxide) levels must be transported to the sensory sites before the ventilatory response can be adjusted. The delay in transfer of partial pressure information from lung to chemosensors depends on cardiac output in general and blood flow rate to the brain in particular. Thus it is important to know how cardiac output and blood flow rates to various tissue centers are controlled. The most of these mathematical models consist of a nonlinear system of delay differential equations with multiple delays [15].

The mathematical model for cardiovascular-respiratory system can be used also for determining the variation of trajectories of some determinant parameters of this system. The behavior of these 
parameters is provided by a qualitative study. The model we are studying was proposed in [1] where two delays are introduced. They consist of delays due of response of pressures to controls of cardiovascular-respiratory system during three cases of physical activity: Walking, Jogging, and Running fast.

This paper is organised as follows. In section 1, we set mathematical model of differentials equations as well as steady state. The section 2 deals with the mathematical model governed by delay differential equations. In two previous sections, the numerical simulations have been done and the test results presented. In section 3 we present general discussions from the numerical results. The concluding remarks are presented in section 4 .

\section{THE ODE MODEL}

The cardiovascular and respiratory system plays a crucial role in controlling the blood flow of human body. The main controls of pressures are heart rate and alveolar ventilation which control the systemic arterial pressures to prevent cardiac accidents [13]. For a healthy subject, it is well known that heart rate and alveolar ventilation depend on his/her level in training during physical activity. A mathematical model developed by S. Timischl-Teschl [5] shows the instability of equilibrium steady. It is governed by many differential equations and it doesn't permit to understand a long-term cardiovascular- respiratory system in the case of aerobic physical activities. A two compartmental mathematical model has been developed in [1] to solve this problem. We consider the functions $P_{v s}$ and $P_{a s}$ as respectively mean blood pressures in systemic arterial region and in systemic venous region. $H$ and $\dot{V}_{A}$ denote the control functions. For a cardiovascular- respiratory system, they design heart rate and alveolar ventilation respectively. The mechanism of this control is not direct and can be represented by outflow functions between systemic arterial and venous compartments that depend on heart rate alveolar ventilation. The model equations arise from straightforward development of mass balance between arterial systemic arterial and systemic venous compartments. For three physical activities (Walking, Jogging and Running fast), the ODE mathematical model is

$$
\left\{\begin{array}{l}
\frac{d P_{a s}(t)}{d t}=-P_{a s}(t)+P_{v s}^{\gamma}(t) f\left(H, \dot{V}_{A}\right) \\
\frac{d P_{v s}(t)}{d t}=-P_{v s}(t)+P_{a s}^{\delta}(t) g\left(H, \dot{V}_{A}\right)
\end{array}\right.
$$

where the constants of the model equations (1) are given as $\gamma=-0.0112, \quad \delta=-0.1724$ and the functions $f$ and $g$ have been identified as follows [1].

- Walking case:

$$
\begin{aligned}
& f\left(H, \dot{V}_{A}\right)=\exp \left(2.6812 \dot{V}_{A}^{-0.0479}+3.4921 H^{-0.0943}\right), \\
& g\left(H, \dot{V}_{A}\right)=\dot{V}_{A} \exp \left(H^{-30.7207}-0.0981\right)
\end{aligned}
$$

- Jogging case:

$$
\begin{aligned}
& f\left(H, \dot{V}_{A}\right)=\exp \left(0.9990 H^{0.1179}+1.1522 H^{0.2280}\right), \\
& g\left(H, \dot{V}_{A}\right)=\dot{V}_{A} \exp \left(H^{-0.2105}-0.0981\right)
\end{aligned}
$$

- Running fast:

$$
\begin{aligned}
& f\left(H, \dot{V}_{A}\right)=\exp \left(0.5472 \dot{V}_{A}^{-0.3820}+0.7518 H^{0.2846}\right), \\
& g\left(H, \dot{V}_{A}\right)=\dot{V}_{A} \exp \left(H^{-0.0985}-1.7440\right)
\end{aligned}
$$

Taking

$$
\begin{gathered}
x(t)=P_{a s}(t), y(t)=P_{v s}(t), \\
u(t)=f\left(H, \dot{V}_{A}\right), v(t)=g\left(H, \dot{V}_{A}\right),
\end{gathered}
$$


then the model (1) becomes

$$
\left\{\begin{array}{l}
\frac{d x(t)}{d t}=-x(t)+y^{\gamma}(t) u(t) \\
\frac{d y(t)}{d t}=-y(t)+x^{\delta}(t) v(t)
\end{array} .\right.
$$

If $X^{*}=\left(x^{*}, y^{*}\right)^{T}$ is an equilibrium point of variable state $(x, y)^{T}$ and $U_{e}=\left(u_{e}, v_{e}\right)^{T}$ is the equilibrium of corresponding to the control parameter $U=(u, v)^{T}$, the steady state values are obtained by solving the following system

$$
\left\{\begin{array}{l}
-x^{*}+\left(y^{*}\right)^{\gamma} u_{e}=0 \\
-y^{*}+\left(x^{*}\right)^{\delta} v_{e}=0
\end{array}\right.
$$

that is

$$
\left\{\begin{array}{l}
\left(y^{*}\right)^{\gamma} u_{e}=x^{*} \\
\left(x^{*}\right)^{\delta} v_{e}=y^{*}
\end{array}\right.
$$

Therefore, the state values are

$$
x^{*}=\left(u_{e}\right)^{\frac{1}{1-\gamma \delta}} \times\left(v_{e}\right)^{\frac{\gamma}{1-\gamma \delta}}
$$

and

$$
y^{*}=\left(u_{e}\right)^{\frac{\delta}{1-\gamma \delta}} \times\left(v_{e}\right)^{\frac{1}{1-\gamma \delta}} .
$$

According to the analysis presented in [1] the steady state $X^{*}$ is stable if when $0<\gamma \delta<1$.

Indeed, to discuss this stability, we consider the linearized system of (2) at $X^{*}$ where the Jacobian matrix is given by

$$
\mathbf{H}=\left[\begin{array}{ll}
-1 & \gamma\left(u_{e}\right)^{\frac{1-\delta}{1-\gamma \delta}}\left(v_{e}\right)^{\frac{\gamma-1}{1-\gamma \delta}} \\
\delta\left(u_{e}\right)^{\frac{\delta-1}{1-\gamma \delta}}\left(v_{e}\right)^{\frac{1-\gamma}{1-\gamma \delta}} & -1
\end{array}\right] .
$$

Then the characteristic equation of the linearized system is

$$
\lambda^{2}+2 \lambda+1-\gamma \delta=0
$$

and this equation has two strictly negative real roots if and only if $0<\gamma \delta<1$.

In numerical simulation, we consider the steady values given in [1] as shown in the table 1 .

Table 1: The rest and steady values of a 30 years old woman during three cases of physical activities for heart rate, alveolar ventilation, venous and arterial systemic pressure.

\begin{tabular}{|c|c|c|c|}
\hline Exercise intensity & Rest & Walking & Jogging \\
\hline Arterial Pas(mmHg) & 104 & 110 & 135 \\
\hline Venous Pvs(mmHg) & 3.566 & 3.46 & 3.28 \\
\hline
\end{tabular}

Numerical simulations given in the figures 1, 2 and 3 show that trajectories of system (1) approach to the steady state. 


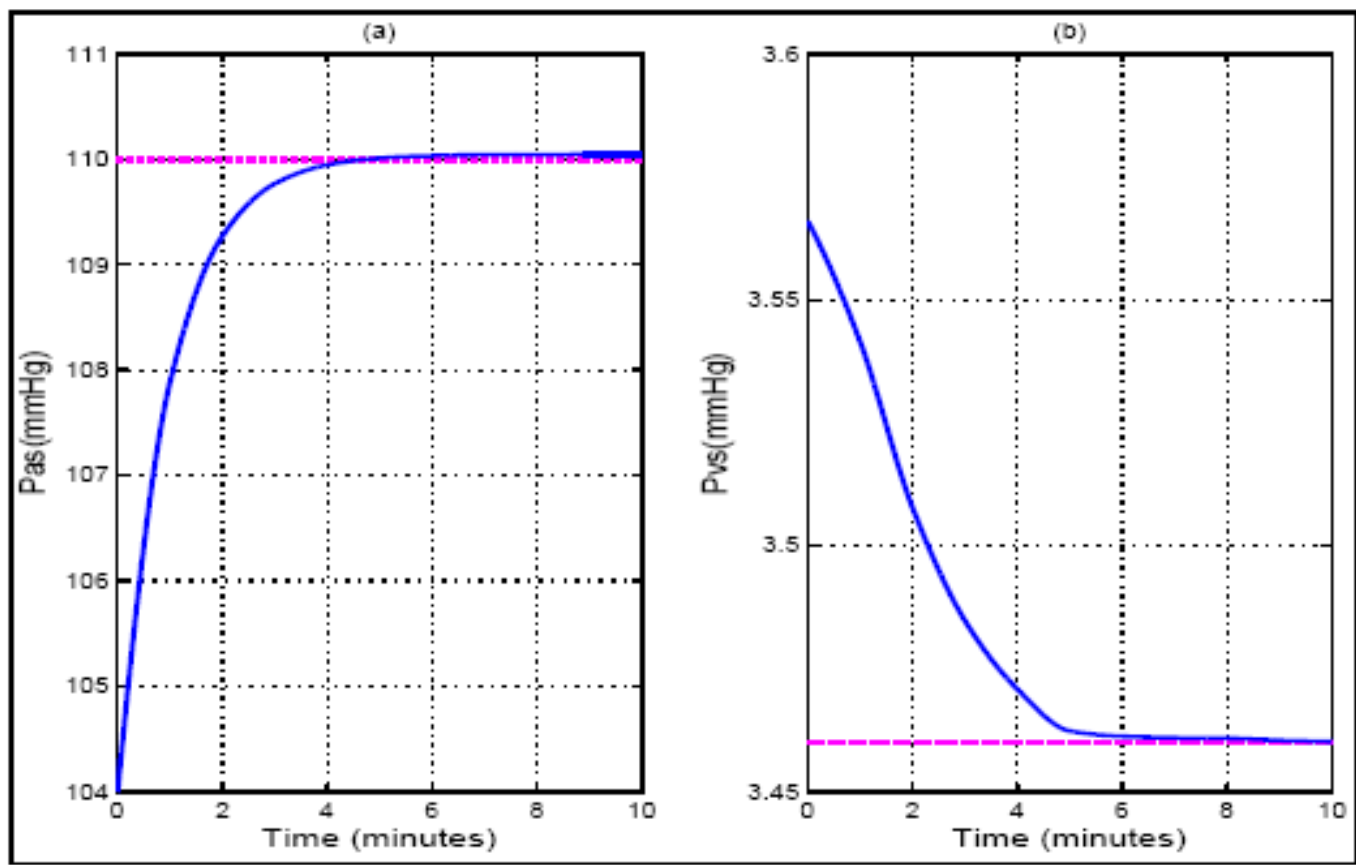

Figure 1: Variation trajectory of systemic arterial (a) and systemic venous (b) pressures for ODE mathematical model. They are compared to their respective steady value (dashed line) for a 30 years old woman during walking physical activity. The simulations are related to the values given in the table 1.

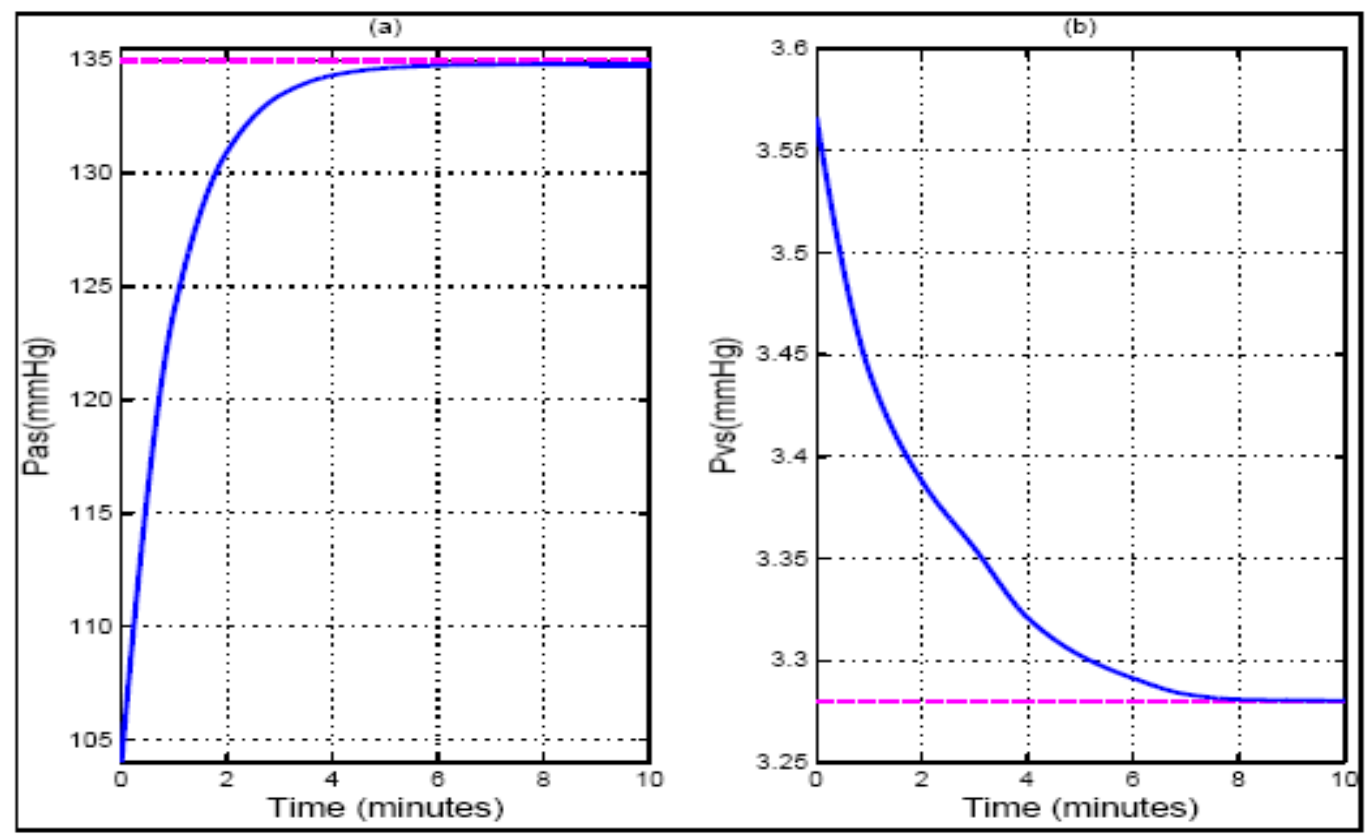

Figure 2: Variation trajectory of systemic arterial (a) and systemic venous (b) pressures for ODE mathematical model. They are compared to their respective steady value (dashed line) for a 30 years old woman during jogging physical activity. The simulations are related to the values given in the table 1. 
A Two Delay Differential Equations Model of Blood Partial Pressures in Human CardiovascularRespiratory System

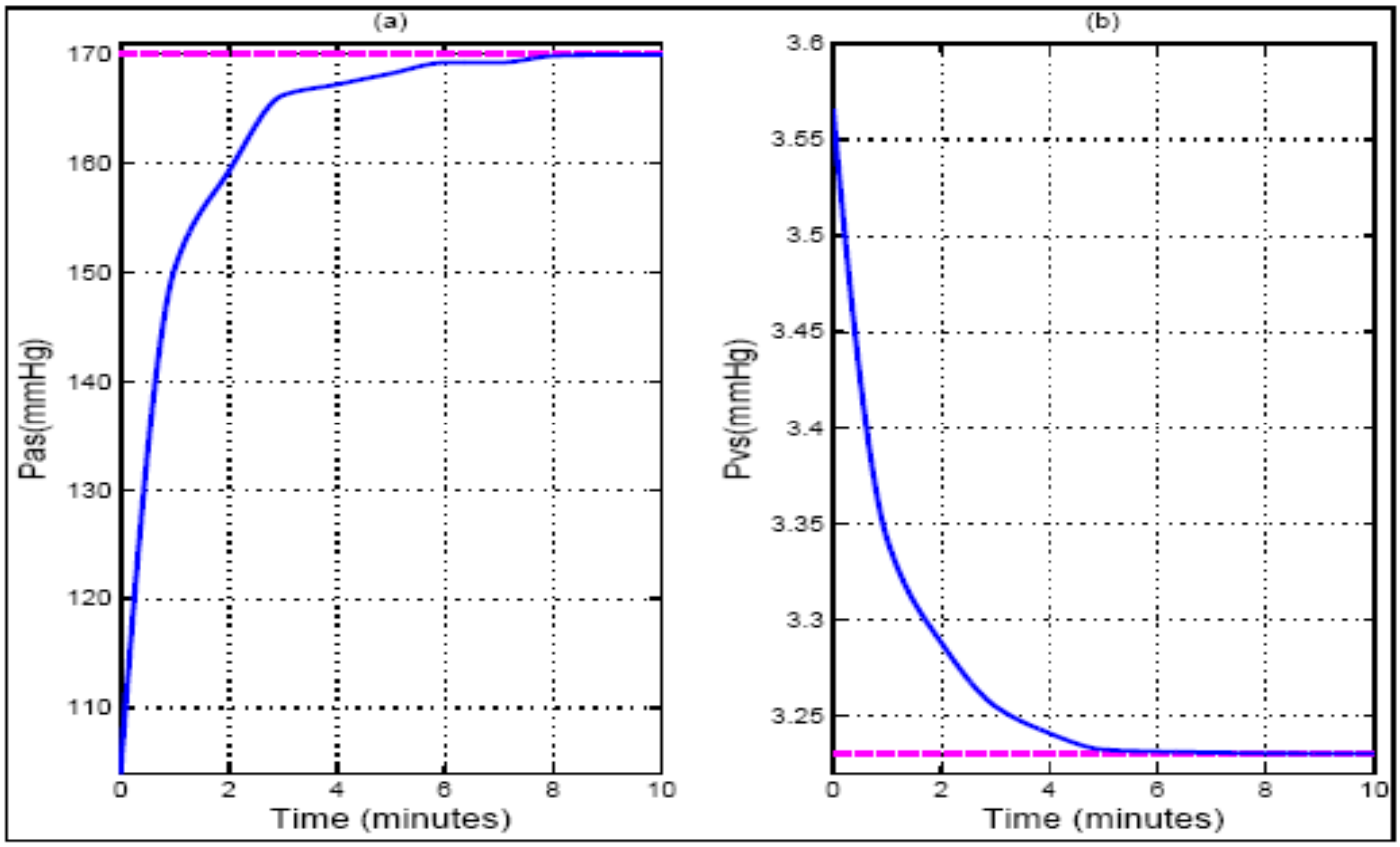

Figure 3: Variation trajectory of systemic arterial (a) and systemic venous (b) pressures for ODE mathematical model. They are compared to their respective steady value (dashed line) for a 30 years old woman during running physical activity. The simulations are related to the values given in the table 1.

\section{The Delay Model}

In this section, we introduce two time delays into system (1) to represent the control phase. The model is given as follows:

$$
\left\{\begin{array}{l}
\frac{d P_{a s}(t)}{d t}=-P_{a s}(t)+P_{v s}^{\gamma}\left(t-\tau_{v s}\right) f\left(H, \dot{V}_{A}\right) \\
\frac{d P_{v s}(t)}{d t}=-P_{v s}(t)+P_{a s}^{\delta}\left(t-\tau_{a s}\right) g\left(H, \dot{V}_{A}\right)
\end{array},\right.
$$

where $\tau_{v s}, \tau_{a s}$ are respectively systemic arterial and systemic venous delays and where the initial values are taken as follows

$$
P_{a s}(\theta)=P_{a s}(0), \quad P_{v s}(\theta)=P_{v s}(0), \quad \theta \in[-\tau, 0] \text { for } \tau=\tau_{a s}+\tau_{v s} .
$$

To study the stability of the steady states $X^{\beta}$, let us define

$$
y_{\tau_{v s}}(t)=y\left(t-\tau_{v s}\right), \quad x_{\tau_{a s}}(t)=x\left(t-\tau_{a s}\right)
$$

the system (6) is written as follows

$$
\left\{\begin{array}{l}
\frac{d x(t)}{d t}=f_{1}\left(x, y_{\tau_{v s}}, u\right)=-x(t)+y_{\tau_{v s}}^{\gamma}(t) u(t) \\
\frac{d y(t)}{d t}=f_{2}\left(y, x_{\tau_{a s}}, v\right)=-y(t)+x_{\tau_{a s}}^{\delta}(t) v(t)
\end{array} .\right.
$$

Taking the first order Taylor series around the equilibrium point, we get 


$$
\left\{\begin{array}{c}
\frac{d x(t)}{d t}=\frac{\partial f_{1}\left(x^{*}, y^{*}, u_{e}\right)}{\partial x}\left(x-x^{*}\right)+\frac{\partial f_{2}\left(x^{*}, y^{*}, u_{e}\right)}{\partial y_{\tau_{v s}}}\left(y_{\tau_{v s}}-y^{*}\right) \\
+\frac{\partial f_{1}\left(x^{*}, y^{*}, u_{e}\right)}{\partial u}\left(u-u_{e}\right) \\
\frac{d y(t)}{d t}=\frac{\partial f_{1}\left(x^{*}, y^{*}, v_{e}\right)}{\partial y}\left(y-y^{*}\right)+\frac{\partial f_{2}\left(x^{*}, y^{*}, v_{e}\right)}{\partial x_{\tau_{a s}}}\left(x_{\tau_{a s}}-x^{*}\right) \\
+\frac{\partial f_{2}\left(x^{*}, y^{*}, v_{e}\right)}{\partial v}\left(v-v_{e}\right)
\end{array}\right.
$$

After calculations, the linearized system becomes

$$
\left\{\begin{array}{l}
\frac{d x(t)}{d t}=-\left(x-x^{*}\right)+\gamma\left(y^{*}\right)^{\gamma-1} u_{e}\left(y_{\tau_{v s}}-y^{*}\right)+\left(y^{*}\right)^{\gamma}\left(u-u_{e}\right) \\
\frac{d y(t)}{d t}=-\left(y-y^{*}\right)+\delta\left(x^{*}\right)^{\delta-1} v_{e}\left(x_{\tau_{a s}}-x^{*}\right)+\left(x^{*}\right)^{\delta}\left(v-v_{e}\right)
\end{array},\right.
$$

which can be written in the following form

$$
\left\{\begin{array}{l}
\frac{d X(t)}{d t}=A_{1} X(t)+A_{2} X\left(t-\tau_{a s}\right)+A_{3} X\left(t-\tau_{v s}\right)+D U(t) \\
X(t)=X_{0},-\tau \leq t \leq 0 .
\end{array}\right.
$$

where we set

$$
\begin{gathered}
X(t)=\left(\begin{array}{c}
x-x^{*} \\
y-y^{*}
\end{array}\right), A_{1}=\left(\begin{array}{cc}
-1 & 0 \\
0 & -1
\end{array}\right), U=\left(\begin{array}{c}
u-u_{e} \\
v-v_{e}
\end{array}\right), \\
A_{2}=\left(\begin{array}{cc}
0 & 0 \\
\delta\left(x^{*}\right)^{\delta-1} v_{e} & 0
\end{array}\right), A_{3}=\left(\begin{array}{cc}
0 & \gamma\left(y^{*}\right)^{\gamma-1} u_{e} \\
0 & 0
\end{array}\right) \text { and } D=\left(\begin{array}{cc}
\left(y^{*}\right)^{\gamma} & 0 \\
0 & \left(x^{*}\right)^{\delta}
\end{array}\right) .
\end{gathered}
$$

The solution of the system (7) can be written as

$\left\{\begin{array}{c}X(t)=K(t) e^{\lambda t} \\ X(0)=K(0)\end{array}\right.$

from where the derivative gives the following equation

$$
\frac{d X(t)}{d t}=\frac{d K(t)}{d t} e^{\lambda t}+\lambda K(t) e^{\lambda t}
$$

Taking into account the equations (8) and (9) the system (7) becomes

$$
\frac{d K(t)}{d t} e^{\lambda t}+\lambda K(t) e^{\lambda t}=A_{1} K(t) e^{\lambda t}+A_{2} K(t) e^{\lambda\left(t-\tau_{a s}\right)}+A_{3} K(t) e^{\lambda\left(t-\tau_{v s}\right)}+D U(t),
$$

that is

$$
\frac{d K(t)}{d t} e^{\lambda t}=\left(A_{1}+A_{2} e^{-\lambda \tau_{a s}}+A_{3} e^{-\lambda \tau_{v s}}-\lambda I\right) K(t) e^{\lambda t}+D U .
$$

We can now find $\lambda$ such that

$$
\left(A_{1}+A_{2} e^{-\lambda \tau_{a s}}+A_{3} e^{-\lambda \tau_{v s}}-\lambda I\right)=0,
$$

thus due to this condition $K(t)$ is the solution: 


$$
\left\{\begin{array}{l}
\frac{d K(t)}{d t} e^{\lambda t}=D U(t) \\
K(0)=X_{0}
\end{array}\right.
$$

where

$$
K(t)=D \int_{0}^{t} U(s) e^{-\lambda s} d s+X_{0}
$$

Consequently from (8) we have

$$
X(t)=e^{\lambda t}\left[D \int_{0}^{t} U(s) e^{-\lambda s} d s+X_{0}\right] .
$$

We know that the stability of this solution depend on the property of the parameter $\lambda$. Therefore, the stability of the solution of equation (7) is obtained using homogeneous equation from the equation of the form

$$
\frac{d X(t)}{d t}=A_{1} X(t)+A_{2} X\left(t-\tau_{a s}\right)+A_{3} X\left(t-\tau_{v s}\right)
$$

that is

$$
\frac{d X(t)}{d t}=0
$$

From the equation (10) we deduce the characteristic equation of the form:

$$
\left|A_{1}+A_{2} e^{-\lambda \tau_{a s}}+A_{3} e^{-\lambda \tau_{v s}}-\lambda I\right|=0 \text {. }
$$

which is written as

$$
\left|\begin{array}{cc}
-1-\lambda & \gamma\left(y^{*}\right)^{\gamma-1}\left(u_{e}\right) e^{-\lambda \tau_{v s}} \\
\delta\left(x^{*}\right)^{\delta-1}\left(v_{e}\right) e^{-\lambda \tau_{a s}} & -1-\lambda
\end{array}\right|=0
$$

After the calculations we get

$$
(-1-\lambda)^{2}-\delta\left(x^{*}\right)^{\delta-1}\left(v_{e}\right) e^{-\lambda \tau_{a s}} \gamma\left(y^{*}\right)^{\gamma-1}\left(u_{e}\right) e^{-\lambda \tau_{v s}}=0,
$$

that is

$$
\lambda^{2}+2 \lambda+1-\delta\left(x^{*}\right)^{\delta-1} \gamma\left(y^{*}\right)^{\gamma-1}\left(v_{e}\right) e^{-\lambda \tau_{a s}}\left(u_{e}\right) e^{-\lambda \tau_{v s}}=0 .
$$

Since from (3) we have

$$
\left(y^{*}\right)^{\gamma}=\frac{x^{*}}{u_{e}} \text { and }\left(x^{*}\right)^{\delta}=\frac{y^{*}}{v_{e}}
$$

we get

$$
\lambda^{2}+2 \lambda+1-\delta \frac{y^{*}}{v_{e}} \frac{1}{x^{*}} \gamma \frac{x^{*}}{u_{e}} \frac{1}{y^{*}}\left(v_{e}\right) e^{-\lambda \tau_{a s}}\left(u_{e}\right) e^{-\lambda \tau_{v s}}=0 .
$$

Finally we obtain the characteristic polynomial of the form

$$
P(\lambda) \equiv \lambda^{2}+2 \lambda+1-\gamma \delta e^{-\lambda\left(\tau_{a s}+\tau_{v s}\right)}=0
$$

that is

$$
P(\lambda) \equiv \lambda^{2}+2 \lambda+1-\gamma \delta e^{-\lambda \tau}=0 .
$$

In [16] is shown that $X^{*}$ is asymptotically stable if all roots of the corresponding characteristic 
equation (13) have negative real parts. However, the comparison with the polynomial characteristic equation (5) for the ODE model allows to say that there is much more difficult to deal with the equation (13) because it is a transcendental equation with infinitely many eigenvalues and the classical Routh-Hurwitz criterion cannot be used to discuss equation (13) anymore. Third justification of difficult to deal with the equation (13) is the existence of some general tests (see [17], for example) that can be used to determine when all eigenvalues of the transcendental equations have negative real parts but to apply such a general test to this specific transcendental equations is very complicated and far from trivial [18].

Using the analytical study, we analyse the distribution of the roots of the transcendental equation (13). Recall that if the relation (4) is satisfied for the parameters $\gamma$ and $\delta$ for the ODE model (1), the steady state $X^{*}$ is stable. This is our starting point so that we shall derive conditions on the parameters to ensure that the steady state of the delay model is still stable.

To proceed, we consider equation (13) with $\tau=\tau_{a s}+\tau_{v s}=0$, that is equation (5). Assuming that all the roots of equation (5) have negative real parts. This is equivalent to the assumption (13). From Rouché's Theorem [19] and the continuity in $\tau=\tau_{a s}+\tau_{v s}$, the transcendental equation (13) has roots with positive real parts if and only if it has purely imaginary roots. We shall determine if (13) has purely imaginary roots, from which we then shall be able to find conditions for all eigenvalues to have negative real parts.

Let us take the eigenvalue of the characteristic equation (13) $\lambda=\eta(\tau)+\omega(\tau)$ where $\eta(\tau)$ and $\omega(\tau)$ depend on the delay $\tau$. Since the equilibrium $X^{\$}$ is stable for the ODE model (1), we deduce that that $\eta(0)<0$ when $\tau=0$. Taking $\tau>0$ but sufficiently small the property of continuity allows to say that $\eta(0)<0$ and $X^{*}$ is stable. Assuming that $\eta\left(\tau_{0}\right)=0$ for certain value of $\odot 0$ such that we have $\lambda=i \omega\left(\tau_{0}\right)$ is a purely imaginary root of (13), then the steady state $X^{*}$ loses its stability and eventually becomes unstable when $\eta(\tau)$ becomes positive. In other words, when $\omega(\tau)$ does not exist, that is, when the characteristic equation (13) does not have purely imaginary roots for all delay, the steady state $X^{*}$ is always stable. We shall show that this indeed is true for the characteristic equation (13). We prove the following result.

\section{Proposition 3.1}

If the relation (4) is satisfied for the parameters $\gamma$ and $\delta$, then the steady state $X^{*}$ of the delay model (6) is absolutely stable; that is, $X^{*}$ is asymptotically stable for all $\tau>0$.

\section{Proof}

$\lambda=i \omega$ is a root of equation (13) if and only if

$$
-\omega^{2}+2 i \omega+1-\gamma \delta e^{-i \omega \tau}=0
$$

that is

$$
-\omega^{2}+2 i \omega+1-\gamma \delta[\cos (\omega \tau)-i \sin (\omega \tau)]=0 .
$$

Separating the real and imaginary parts, we obtain

$-\omega^{2}+1-\gamma \delta \cos (\omega \tau)=0$

and

$2 \omega+\gamma \delta \sin (\omega \tau)=0$.

Thus we have

$\gamma \delta \cos (\omega \tau)=1-\omega^{2}$ 
and

$$
\gamma \delta \sin (\omega \tau)=-2 \omega \text {. }
$$

Adding up the squares of both two last equations, we obtain

$$
(\gamma \delta)^{2}=\left(1-\omega^{2}\right)^{2}+(-2 \omega)^{2} .
$$

After calculation, we have

$\gamma \delta=1+\omega^{2}$.

Since the relation (4) is satisfied, we then have

$$
0<1+\omega^{2}<1 .
$$

Taking the right inequality, we obtain

$$
\omega^{2}<0
$$

which does not have mathematically the meaning. This implies that there is no $\omega$ such that $i \omega$ is an eigenvalue of the characteristic equation (13). Therefore, the real parts of all the eigenvalues of (13) are negative for all delay $\tau>0$ and $X^{*}$ is asymptotically stable In numerical simulation, we consider again constants given in the table 1 . The values of delays $\tau_{a s}$ and $\tau_{v s}$ are given in the table 2 .

Table 2: Delay parameters in the walking, jogging and running cases.

\begin{tabular}{|l|l|l|l|}
\hline Delay parameters & Walking & Jogging & Running \\
\hline$\tau_{\text {as }}$ & 0.6689 & 0.6698 & 0.6989 \\
\hline$\tau_{\mathrm{vs}}$ & 0.6889 & 0.6994 & 0.6999 \\
\hline
\end{tabular}

The figures 4, 5 and 6 are results obtained from the numerical simulations of the system (6). They show that the steady state $X^{*}$ is asymptotically stable. Compared with the 1,2 and 3, we can see that though the delay causes transient oscillations in the components, the steady state $X^{*}$ is still stable.

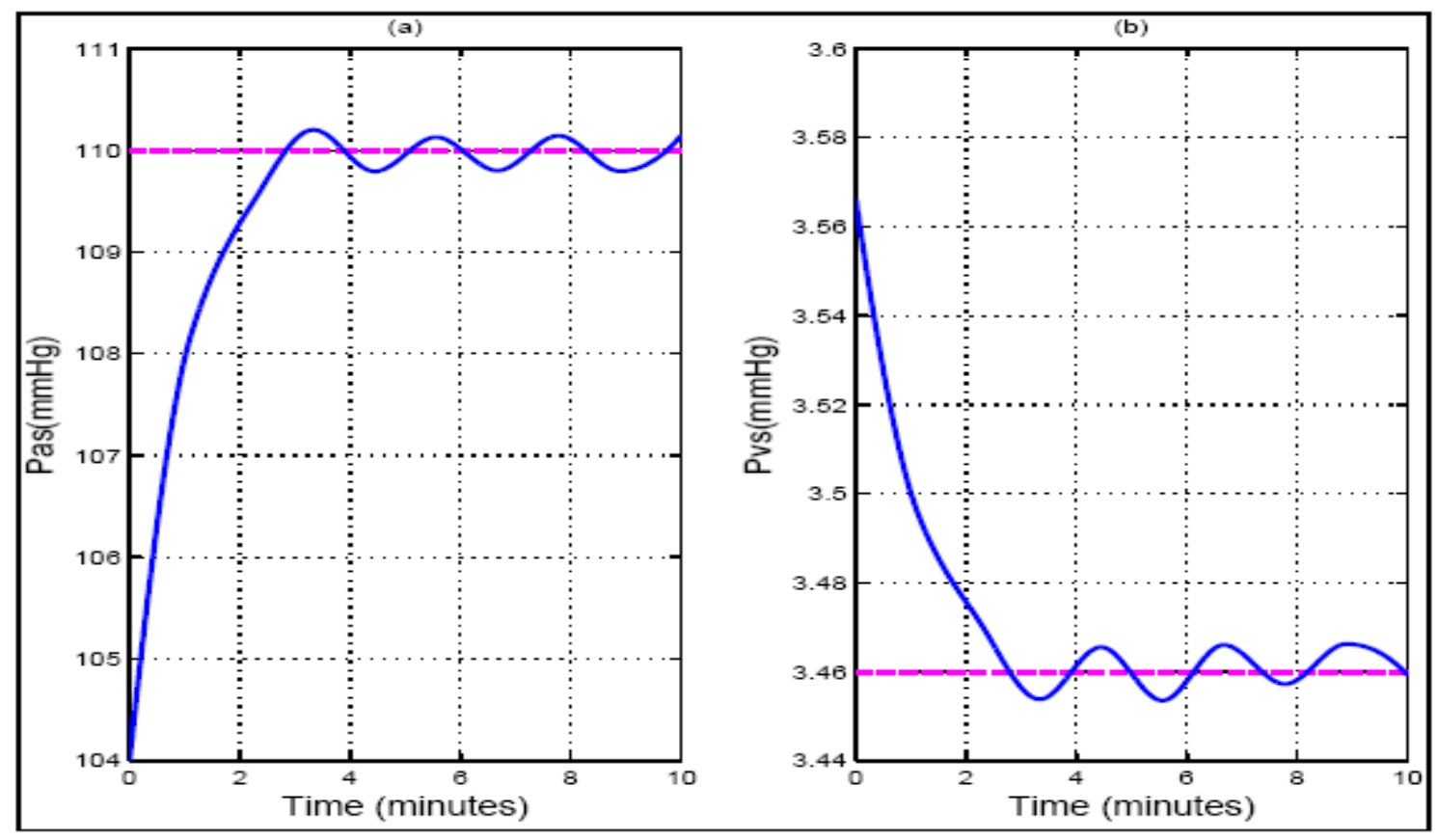

Figure 4: Variation trajectory of systemic arterial (a) and systemic venous (b) pressures for delay mathematical model. They are compared to their respective steady value (dashed line) for a 30 years old woman during walking physical activity. The simulations are related to the values given in the table 1 and the values of delay are presented in the table 2. 


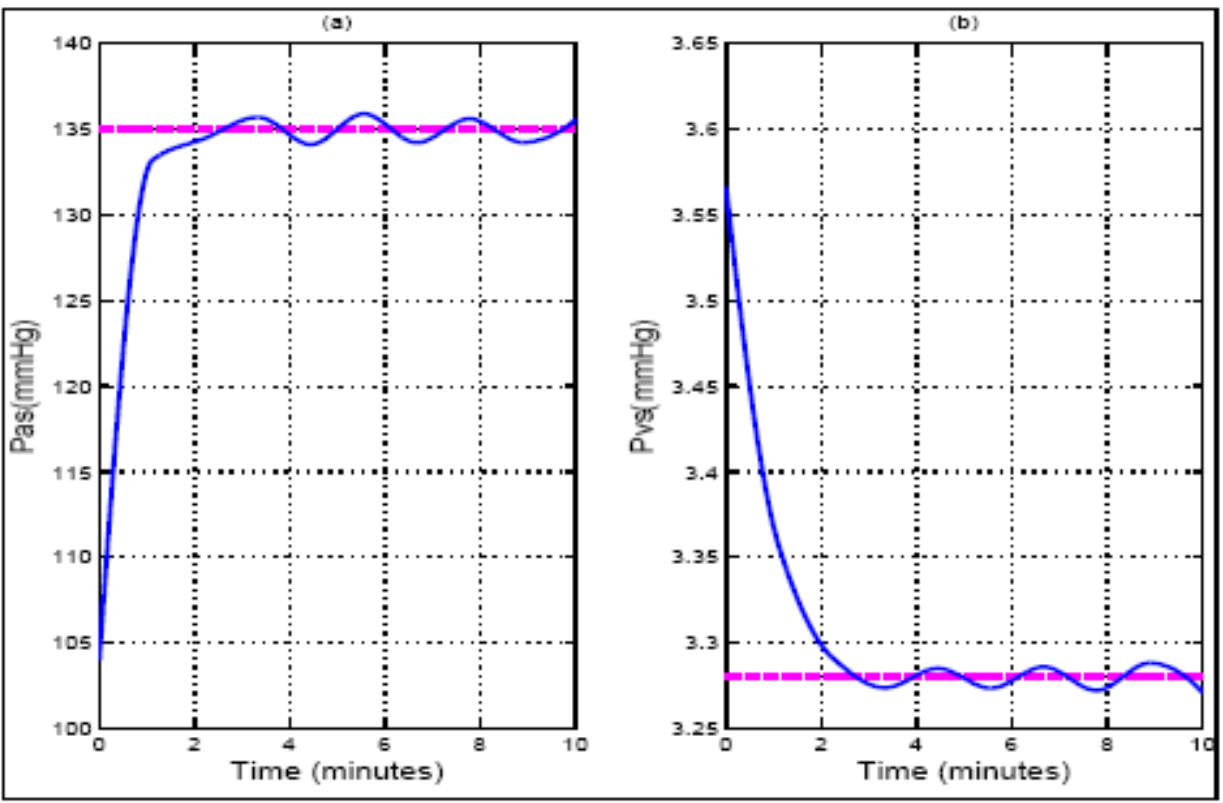

Figure 5: Variation trajectory of systemic arterial (a) and systemic venous (b) pressures for delay mathematical model. They are compared to their respective steady value (dashed line) for a 30 years old woman during jogging physical activity. The simulations are related to the values given in the table 1 and the values of delay are presented in the table 2.

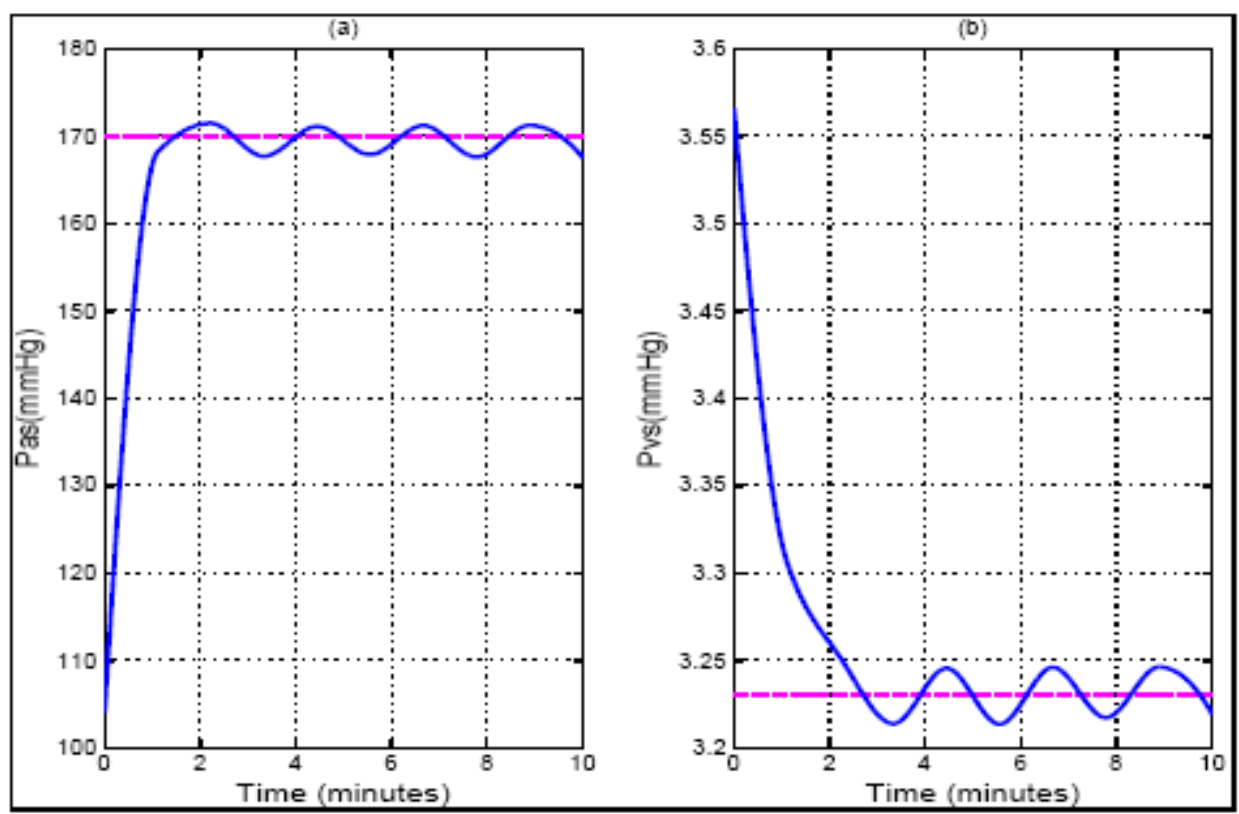

Figure 6: Variation trajectory of systemic arterial (a) and systemic venous (b) pressures for delay mathematical model. They are compared to their respective steady value (dashed line) for a 30 years old woman during running physical activity. The simulations are related to the values given in the table 1 and the values of delay are presented in the table 2.

\section{Remark 3.2}

According to the Proposition 3.1, the steady state of the delay model () is asymptotically stable for all delay values. This means that the stability is independent of the delay. However, we should point out that if the conditions of the Proposition 3.1 are not satisfied, then the stability of the steady state depends on the delay value and the delay could even induce oscillations.

Let us be interested in one example to clarify the idea of Remark 3.2. If we take $\gamma \delta>1$ then from equation (18) has a positive root $y_{0}$. This implies that the characteristic equation (13) has a pair of purely imaginary roots $\pm i \omega_{0}$. 
Let $\lambda(\tau)=\eta(\tau)+i \omega(\tau)$ be the eigenvalue of equation () such that $\eta\left(\tau_{0}\right)=0$ and $\omega\left(\tau_{0}\right)=\omega_{0}$. From (16) and (17) we have

$$
\tan \omega_{0} \tau=\frac{2 \omega_{0}}{\omega_{0}^{2}-1}
$$

then

$$
\tau_{j}=\frac{1}{\omega_{0}} \arctan \left(\frac{2 \omega_{0}}{\omega_{0}^{2}-1}\right)+\frac{j \pi}{\omega_{0}}, j=0,1,2, \ldots
$$

It is also easy to verify the the following transversality condition:

$$
\left.\frac{d}{d \tau} \operatorname{Re} \lambda(\tau)\right|_{\tau=\tau_{0}}=\left.\frac{d}{d \tau} \eta(\tau)\right|_{\tau=\tau_{0}} .
$$

The continuity allows us to say that real part of $\lambda(\tau)$ becomes positive when $\tau>\tau_{0}$ and the steady state $X^{*}$ becomes unstable. Moreover, the behavior of $X^{*}$ is discussed in [20] from where a we conclude that Hopf bifurcation occurs when $c$ passes through the critical value $\tau_{0}$.

The above analysis can be summarized into the following proposition.

\section{Proposition 3.3}

Suppose that $\gamma \delta>1$ is satisfied, then the infected steady state $X^{*}$ of the delay model () is asymptotically stable when $\tau<\tau_{0}$ and unstable when $\tau>\tau_{0}$, where

$$
\tau_{0}=\frac{1}{\omega_{0}} \arctan \left(\frac{2 \omega_{0}}{\omega_{0}^{2}-1}\right)
$$

When $\tau=\tau_{0}$, a Hopf bifurcation occurs; that is, a family of periodic solutions bifurcates from $X^{*}$ as s passes through the critical value $\tau_{0}$.

\section{DISCUSSION}

The mathematical model for cardiovascular-respiratory system incorporating a time delay has been used (See par example [21]). Now the challenge is to determine how the delay of cardiovascularrespiratory affects overall determinant parameters and, mathematically, how the delay effects the dynamics of systems.

We have used the mathematical model proposed in [1]. It is governed by a system of two differential equations. Using stability analysis we obtained sufficient conditions on the parameters for the stability. For parameter where the condition (4) is satisfied allows the stability of steady state and numerical simulations confirmed the analysis. The variation of determinant parameters of cardiovascular respiratory system are due to the variation of its controls (Heart rate and alveolar ventilation). Consequently, these parameters reach the steady value and stay around is due the increase of controls to their equilibrium values.

We then introduced two time delays into the model which describes the time between controls and the reaction of pressures of cardiovascular-respiratory system. By analyzing the transcendental characteristic equation, we analytically derived stability conditions for the steady state in terms of the parameters and independent of the delay. Using the parameter values in tables 1 and 2, we found that all the conditions are satisfied. Thus, the steady state is stable, independent of the size of the delay, though the time delay does cause transient oscillations in all components. Numerical simulations confirmed our analysis. Biologically, it implies that the delay in cardiovascular-respiratory system can cause the controls and pressures to fluctuate at the beginning of physical activity and in a longer term determinant of cardiovascular-respiratory system converge to the their equilibrium values due to controls. Under another set of assumptions on the parameters, the stability of the steady state depends on the delay and even delay-induced oscillations could occur via instability. 


\section{CONCLUding Remarks}

We have investigated in this work a bi-compartmental mathematical model of ordinary differential and delay differential equations for blood partial pressures in human cardiovascular-respiratory system. Numerical simulations of the model illustrate the responses of arterial and venous pressures of cardiovascular-respiratory system due to its controls (heart rate and alveolar ventilation). The qualitative analysis of the model shows that the steady state is stable though the delays can cause transient oscillations around the equilibrium value. The numerical simulations confirmed the analytical analysis for a 30 years old woman during three different physical activities which are walking, jogging and running.

\section{REFERENCES}

[1] Jean Marie Ntaganda and Benjamin Mampassi, Modelling blood partial Pressures of the human cardiovascular respiratory system, Applied Mathematics and computation, Vol.187, pp 11001108, 2007.

[2] J.T Ottesen, Modeling of the baroreflex-feedback mechanism with time delay, J.Math.Bio, 36(1), pp. 41-63.

[3] Ellwein L. M., Cardiovascular and Respiratory Regulation, Modeling and Parameter Estimation, North Carolina State University, 2008.

[4] Kaufman A., Development of a Fast Response Carbon Monoxide Sensor for Respiratory Gas Analysis, University College London, 2001.

[5] S. Timischl, A global Model for the Cardiovascular and Respiratory System, PhD thesis, KarlFranzens-Universit of Graz, August 1998.

[6] Valdessa A. A., Heart Rate Regulation: Modeling and Analysis, Raleigh, North Carolina University, 2009.

[7] Fishman A. P., editor, The respiratory system, Volume 3:II: 2 of Handbook of Physiology, American Physiological Society, Bethesda, MD, 1983.

[8] S. Timischl-Teschl, Modeling the human Cardiovascular-Respiratory Control System: An optimal Control Application to the Transition to Non-REM Sleep, Mathematical Biosciences and engineering - Volume 7, Number 07, 2004.

[9] F. Kappel, S. Lafer, R.O. Peer, A model for the cardiovascular system under an ergometric workload, Surv. Math. Ind. 7, 239--250, 1997.

[10] F. Kappel, and R. O. Peer, A mathematical model for fundamental regulation processes in the cardiovascular model, J. Math. Biol. 31(6), pp. 611- 631, 1993.

[11] M. Kuebler,M. Mertens and R. Axel, A two component simulation model to teach respiratory mechanics, Advance Physiology Education 31,218-222, 2007

[12] S. Ganzert, K. Moller, Kristian, L. D. Readt and J. Guttmann, Equation discovery for model identification in respiratory mechanics under condition of mechanical ventilation, ICML07 USA, 24June 2007.

[13] S. Sepehris, Physical model of human respiration, Young Researchers club, Islamic Azad university of Shiraz, 12-17, 2007.

[14] Horgan, J.D. and Lange, R.L, Digital computer simulation of the human respiratory system, IEEE International Conference Record, pages 149-157, 1963.

[15] J. J. Batzel and H. T. Tran, Modeling instability in the control system for human respiration: applications to infant non-REM sleep, Applied Mathematics and Computation, Volume 110 Issue 1, April 2000, Pages 1-51

[16] R. Bellman, K.L. Cooke, Differential-Difference Equations, Academic Press, New York, 1963.

[17] G. Stépán, Retarded Dynamical Systems: Stability and Characteristic Functions, Longman, UK, 1989.

[18] R.V. Culshaw, Mathematical Models of Cell-to-Cell and Cell-Free Viral Spread of HIV Infection, MSc Thesis, Dalhousie University, Halifax, Canada, 1997.

[19] J. Dieudonné, Foundations of Modern Analysis, Academic Press, New York, 1960.

[20] B.D. Hassard, N.D. Kazarinoff, Y.H. Wan, Theory and Applications of Hopf Bifurcation, 

Respiratory System

Cambridge University, Cambridge, 1981.

[21] J. M. Ntaganda, R. Muremyi, Hopf Bifurcation of A Mathematical Model of Blood Partial Pressures in Human Cardiovascular-Respiratory System, International Journal of Scientific and Innovative Mathematical Research (IJSIMR), Volume 2, Issue 7, July 2014, PP 656-667.

\section{AUTHOR's BIOGRAPHY}

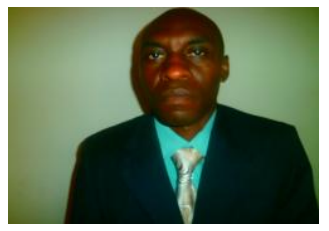

Prof. Ntaganda Jean Marie, PhD holder in Numerical Analysis from Cheikh Anta Diop University of Dakar, Senegal (2004-2006) and Ouagadougou University, Burkina Faso (2004-2007). He is Associate Professor in Applied Mathematics at University of Rwanda, College of Science and Technology,

School of Sciences, Department of Mathematics where he is module leader of computation modules including Numerical Analysis and Programming. Since 2005 he is active researcher in Biomathematics where at least 30 publications have been published in international peer-reviewed journals. 